\title{
Comparative multiplex dosage analysis in spinocerebellar ataxia type 2 patients
}

\author{
F. Calì ${ }^{1}$, V. Chiavetta ${ }^{1}$, A. Ragalmuto ${ }^{1}$, M. Vinci ${ }^{1}$, G. Ruggeri ${ }^{1}$, \\ P. Schinocca ${ }^{1}$ and V. Romano ${ }^{1,2}$ \\ ${ }^{1}$ Laboratory of Molecular Genetics, IRCCS Associazione Oasi Maria SS, \\ Troina, Italy \\ ${ }^{2}$ Department of Physics and Chemistry, University of Palermo, Palermo, Italy \\ Corresponding author: V. Romano \\ E-mail: valentino.romano@unipa.it
}

Genet. Mol. Res. 12 (2): 1176-1181 (2013)

Received July 17, 2012

Accepted November 13, 2012

Published April 12, 2013

DOI http://dx.doi.org/10.4238/2013.April.12.4

\begin{abstract}
We developed a new application of comparative multiplex dosage analysis (CMDA) for evaluation of the ataxin 2 gene. Expansions of the triplet $\mathrm{CAG}$ can cause spinocerebellar ataxia type 2 (SCA2), a neurodegenerative disease with an autosomal-dominant mode of inheritance. Molecular diagnosis of SCA2 is routinely based on the use of conventional PCR to detect the CAG expansion. However, PCR does not amplify an allele with an expansion of many triplets $(>80)$, which is typically found in infantile and juvenile forms of SCA2, thus leading to false negatives. We propose the analysis of the ATXN2 gene by CMDA to complement existing methods currently used for the detection of large expansions of the CAG repeat. Using CMDA, the presence of any longer mutated allele in a heterozygous patient or fetus would be inferred due to dosage variation of the very frequent normal allele \#22. CMDA can be completed in 1 day, at very low cost, and would be a useful tool for prenatal diagnosis and for diagnosis of presymptomatic forms of early-onset SCA2.
\end{abstract}

Key words: Ataxin 2; Spinocerebellar ataxia type 2; Quantitative PCR; Autosomal dominant; Prenatal diagnosis 


\section{INTRODUCTION}

Spinocerebellar ataxia type 2 (SCA2), a severe neurodegenerative disorder transmitted by an autosomal dominant mode of inheritance, is caused by expansion of a CAG repeat in exon 1 of the ataxin 2 (ATXN2) gene. Expansion of this CAG repeat below a threshold of 32 (range, 14-31) is present in (unaffected) individuals of the general population with the 22-repeat allele occurring at a frequency of about $90 \%$ (Sobczak and Krzyzosiak, 2005). There is a gray zone in the range of 32-34 CAG repeats for which the clinical outcome is uncertain, whereas 35-500 repeats are fully penetrant (Sequeiros et al., 2010). Interestingly, there is an inverse correlation between the number of repeats, in the pathological range, and the age of onset of the disease (Schols et al., 1997). Specifically, larger expansions (from 130 to more than 200 CAG repeats) are detected in rare patients with the infantile and juvenile forms of SCA2 (Babovic-Vuksanovic et al., 1998; Mao et al., 2002; Moretti et al., 2004), whereas the more common smaller expansions (between 34 and 57 CAG repeats) are detected in patients with the late-onset forms of the disease appearing in adult life, by the age of about 20-40 years (Moretti et al., 2004). As it is expected for a dynamic mutation, the larger expansions of CAG repeats seen in the early forms of SCA2 originate as aberrant gametogenesis events occurring in one of the two parents bearing a repeat number in the ATXN2 gene typical of the late-onset forms (Mao et al., 2002).

An accurate diagnosis of SCA2 strongly relies on the use of molecular genetic tests that are all based on polymerase chain reaction (PCR). Conventional PCR in conjunction with gel electrophoresis (PCR-CAG) (Pulst et al., 1996) represents an effective and simple assay that is routinely used to detect $\mathrm{CAG}$ expansions in adult patients, in presymptomatic subjects ( $<80$ CAG repeats). A limitation of this test is that allele expansions above 80 repeats will not be detected, thus leading to false negatives in the diagnosis of the early forms of SCA2. This problem appears to be especially acute in prenatal diagnosis as well as for detecting presymptomatic children. To overcome this limitation, new methods have been developed such as the fluorescent repeat-primed PCR assay (STR-PCR) (Cagnoli et al., 2006) and the use of Southern blotting and hybridization (Mao et al., 2002). In line with these studies, we proposed the use of comparative multiplex dosage analysis (CMDA) to assess the ATXN2 genotype.

\section{MATERIAL AND METHODS}

Genomic DNA was isolated from peripheral blood lymphocytes using the salt chloroform extraction method. Informed consent was obtained from each research subject. Quantification of DNA extracted from each sample was done using the Spectrophotometer Nanodrop ND-1000 (Thermo Scientific, USA) $(260 / 280 \mathrm{~nm}$ absorbance ratio of $\sim 1.8 ; 260 / 230 \mathrm{~nm}$ ratio in the range of 2.0-2.2). DNA in the solution was quantified (260 $\mathrm{nm}$ absorbance) and checked for degradation on an agarose gel. We genotyped 8 SCA2 patients and 27 unaffected blood donors from the general population. SCA2 patients were from several Italian academic and non-academic clinical centers, previously referred to our laboratory (Laboratory of Molecular Genetics at Oasi Institute, Troina, Italy) for genetic testing. The 27 neurotypical blood donors were from the Sicilian population (Italy). We first performed the SCA2 CAG repeat routine standard genetic test (PCR-CAG) as described by Pulst et al. (1996). Second, patients and controls were retested according to Cagnoli et al. (2006). Aliquots of the PCR products were then sized using an ABI3130 capillary electrophoresis apparatus. 
CMDA was performed as described in our previous paper (Calì et al., 2010). Briefly, CAG repeats of the SCA2 gene were co-amplified by fluorescent multiplex PCR with primers designed on specific regions of the genes encoding myelin protein zero (PROZF), neuroligin 3 (NLGN), MutL homologue 1 (MLH1), and phenylalanine hydroxylase (PAH) used as controls. Primer sequences, FAM-labeled, sizes of the PCR products, and references are shown in Table 1. PCR mix contained 200-300 ng genomic DNA and 10, 30, 30, 20, and 30 pmol primers for the PROZF, NLGN, PAH, SCA2, and MLH1 genes, respectively. Twenty PCR cycles were used to ensure the reaction and were kept within the exponential phase. The multiplex reaction contained 2.5 U GoTaq DNA polymerase (Promega, Madison, WI, USA), $1.25 \mathrm{mM}$ of each dNTP and 5\% DMSO (1\% final concentration) in a final reaction mixture of $50 \mu \mathrm{L}$. The PCR conditions were $94^{\circ} \mathrm{C}$ for $5 \mathrm{~min}$, followed by 20 cycles of $94^{\circ} \mathrm{C}$ for $40 \mathrm{~s}, 59^{\circ} \mathrm{C}$ for $40 \mathrm{~s}$, and $72^{\circ} \mathrm{C}$ for $1 \mathrm{~min}$ and $30 \mathrm{~s}$, and finally 1 cycle of $72^{\circ} \mathrm{C}$ for $7 \mathrm{~min}$. The PCR products were resolved on an ABI3130 (Applied Biosystems, Foster City, CA, USA) sequence analyzer according to manufacturer instructions. In particular, $2 \mu \mathrm{L}$ PCR product was mixed with 12 $\mu \mathrm{L}$ formamide and $1 \mu \mathrm{L}$ Genescan 500 ROX size standard (Applied Biosystems). The mixture was denatured at $96^{\circ} \mathrm{C}$ for $3 \mathrm{~min}$ and placed on ice until further analysis. Capillary electrophoresis was performed using POP7 gel, filter set D and a $36 \mathrm{~cm}$ column. Fragment sizes and peak areas were determined by the GeneMapper 3.7 software (Applied Biosystems).

Table 1. PCR primer sequences for comparative multiplex dosage analysis.
\begin{tabular}{lllcl}
\hline Primer & Forward FAM-labeled sequence (5'-3') & Reverse sequence (5'-3') & Product size (bp) & Reference \\
\hline PAH & TGGCTTAAACCTCCTCCCC & CTGGGCTCAACTCATTTGAG & 189 & Cali et al., 2010 \\
PROZF & CAGTGGACACAAAGCCCTCTGTGTA & GACACCTGAGTCCCAAGACTCCCAG & 389 & Rowland et al., 2001 \\
MLH1 & GTAGTCTGTGATCTCCGTT & ATGTATGAGGTCCTGTCCTA & 244 & Saugier-Veber et al., 2001 \\
NLGN & CCTGAGGATGGTGTCTCTGGCA & CAGTGGGGCCTACCATAGGAAC & 361 & Cali et al., 2010 \\
SCA2 & GGGCCCCTCACCATGTCG & CGGGCTTGCGGACATTGG & 130 (allele \#22) & Pulst et al., 1996 \\
\hline
\end{tabular}

Results from the samples (patients with suspected SCA) were compared with those from control individuals. The CMDA data were efficiently processed using a spreadsheet generated in Microsoft Excel. First, the data corresponding to each sample (patient's and control's DNAs) were normalized by dividing each probe's signal strength (i.e., the area of each peak) by the average signal strength obtained for the 3 autosomal control probes (PROZF, MLH1 and PAH) to generate for each peak a relative peak area $\left(\mathrm{RPA}_{1}\right)$ value. The RPA value for each probe in each sample was then compared to that of at least three female control samples by dividing, for each peak, the sample RPA $A_{1}$ by the female control RPA to yield the RPA $A_{2}$ value. The $\mathrm{RPA}_{2}$ ratio was then used to define the following categories: i) $\sim 1$, for the two copies, ii) $\sim 0.5$ for one copy. The sensitivity of this gene-dosage test was verified by comparing the RPA between males $\left(\mathrm{RPA}_{2} \sim 0.5\right)$ and females $\left(\mathrm{RPA}_{2} \sim 1\right)$ using the X-linked NLGN gene as control. Other details on normalizing and quality test are described by Calì et al. (2010).

\section{RESULTS AND DISCUSSION}

Eight patients and 27 normal subjects were first analyzed by the PCR-CAG test (Pulst et al., 1996), and the results were confirmed by STR-PCR (Cagnoli et al., 2006) (data not shown). For gene dosage analysis by the CMDA test, we chose the 22-repeat allele due to its high frequency $(>90 \%)$. 
CMDA carried out in these 35 subjects confirmed the presence of one and two 22-repeat alleles for the 11 heterozygotes (expected $\mathrm{RPA}_{2}=0.5$; observed $\mathrm{RPA}_{2}=0.46 ; \mathrm{SD} \pm 0.05$ ) and 24 homozygotes (expected $\mathrm{RPA}_{2}=1$; observed $\mathrm{RPA}_{2}=1.08 ; \mathrm{SD} \pm 0.06$ ), respectively (see Table 2 and Figure 1). The accuracy of the CMDA test to discriminate 1 copy vs 2 copies was checked using the X-linked NLGN gene in 14 males (expected $\mathrm{RPA}_{2}=0.5$; observed $\mathrm{RPA}_{2}=0.56$; $\mathrm{SD}$ \pm 0.06 ) and 21 females (expected $\mathrm{RPA}_{2}=1$; observed $\mathrm{RPA}_{2}=1.1 ; \mathrm{SD} \pm 0.093$ ). The above $\mathrm{SD}$ values indicated that the inter-sample variability in the CMDA was very low.

Table 2. Genotypes of the ATXN2 gene detected in SCA2 patients and normal individuals.

\begin{tabular}{lccc}
\hline Subjects & Number of subjects tested & PCR-CAG & CMDA (No. copies/allele \#22) \\
\hline Controls & 24 & $22 / 22$ & 2 \\
& 3 & $22 / 23$ & 1 \\
Patients & 1 & $22 / 72$ & 1 \\
& 2 & $22 / 40$ & 1 \\
& 1 & $22 / 41$ & 1 \\
& 2 & $22 / 38$ & 1 \\
\hline
\end{tabular}

$\mathrm{CMDA}=$ comparative multiplex dosage analysis.
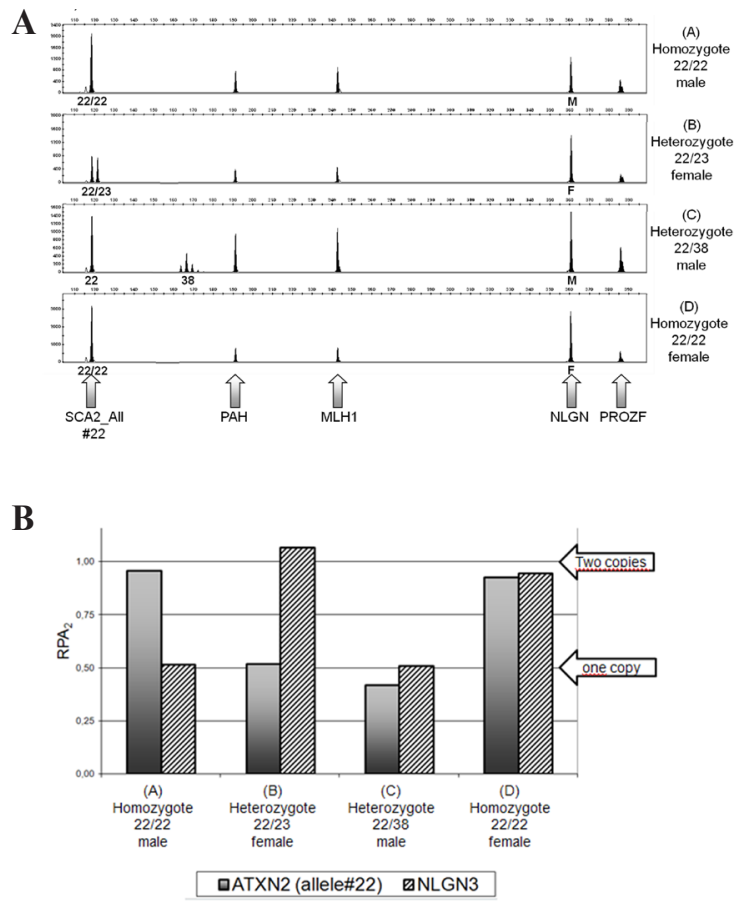

Figure 1. Comparative multiplex dosage analysis of the ATXN2 gene in SCA2 patients and control subjects. A. Electropherograms of 4 typical experiments $(C=$ patient; A, B and $\mathrm{D}=$ controls $)$ showing DNA fragments corresponding to the CAG repeat of the ATXN2 gene co-amplified with specific regions of the X-linked NLGN gene and several control genes (PROZF, NLGN, PAH). B. The histogram displays the plot of RPA, ratios (see text for explanation) computed for ATXN2 (allele \#22) and NLGN genes for each of the 4 subjects tested (A-D) described above. An RPA 2 value of $\sim 1$ corresponds to 2 copies of allele \#22 of the ATXN gene in homozygotes or 2 copies of the NLGN gene in females. An RPA value of $\sim 0.5$ corresponds to 1 copy of allele \#22 of the ATXN gene in heterozygotes or 1 copy of the NLGN gene in males. 
CMDA has been used to identify dosage variation of whole genes as well as exon deletions or duplications (Saugier-Veber, 2001; Calì et al., 2010). In this paper, we show a new application of CMDA in the analysis of mutations of the ATXN2 gene causing SCA2, a severe neurodegenerative disorder transmitted by an autosomal dominant mode of inheritance. Using this method, we showed that by gene dosage analysis it is possible to discriminate between homozygotes and heterozygotes who bear 2 copies and 1 copy, respectively, of the very frequent 22-repeat allele. Using this approach, the presence of any longer mutated allele (even the $>80$ CAG repeats) in a heterozygous patient or fetus would be inferred from dosage variation in the normal allele.

We think that this test is most suitable for prenatal diagnosis and presymptomatic diagnosis of the juvenile/infantile forms of SCA2. Although rare, these forms may appear in sibs from one affected or presymptomatic parent with the late-onset form of SCA2 as an effect of a triplet expansion occurring in the parental gametogenesis due to the dynamic nature of the CAG repeat (Richards, 2001; Mao et al., 2002).

We also took into account the possibility that a copy number variant (CNV), specifically a deletion, would overlap the ATXN2 gene. In such an event, a CNV loss would be wrongly interpreted as an expansion (false positive). However, we consider such a possibility very unlikely since in a search performed in the Database of Genomic Variants (website: http://projects.tcag.ca/variation/) containing CNV data for about 500,000 neurotypical individuals of the general population, we did not identify any CNV overlapping the SCA2 gene.

In summary, the method we developed can be used in conjunction with existing methods to confirm the presence of an expanded allele in patients with both the early- and late-onset forms of SCA2, and in principle, it may be used for the detection of repeat expansions in other disorders.

\section{ACKNOWLEDGMENTS}

Research supported by the Italian Ministry of Health: Current Research 2012 and "5 per thousand" funding.

\section{REFERENCES}

Babovic-Vuksanovic D, Snow K, Patterson MC and Michels VV (1998). Spinocerebellar ataxia type 2 (SCA 2) in an infant with extreme CAG repeat expansion. Am. J. Med. Genet. 79: 383-387.

Cagnoli C, Stevanin G, Michielotto C, Gerbino PG, et al. (2006). Large pathogenic expansions in the SCA2 and SCA7 genes can be detected by fluorescent repeat-primed polymerase chain reaction assay. J. Mol. Diagn. 8: 128-132.

Calì F, Ruggeri G, Vinci M, Meli C, et al. (2010). Exon deletions of the phenylalanine hydroxylase gene in Italian hyperphenylalaninemics. Exp. Mol. Med. 42: 81-86.

Mao R, Aylsworth AS, Potter N, Wilson WG, et al. (2002). Childhood-onset ataxia: testing for large CAG-repeats in SCA2 and SCA7. Am. J. Med. Genet. 110: 338-345.

Moretti P, Blazo M, Garcia L, Armstrong D, et al. (2004). Spinocerebellar ataxia type 2 (SCA2) presenting with ophthalmoplegia and developmental delay in infancy. Am. J. Med. Genet. A 124A: 392-396.

Pulst SM, Nechiporuk A, Nechiporuk T, Gispert S, et al. (1996). Moderate expansion of a normally biallelic trinucleotide repeat in spinocerebellar ataxia type 2. Nat. Genet. 14: 269-276.

Richards RI (2001). Dynamic mutations: a decade of unstable expanded repeats in human genetic disease. Hum. Mol. Genet. 10: 2187-2194.

Rowland JS, Barton DE and Taylor GR (2001). A comparison of methods for gene dosage analysis in HMSN type 1. $J$. Med. Genet. 38: 90-95. 
Saugier-Veber P, Drouot N, Lefebvre S, Charbonnier F, et al. (2001). Detection of heterozygous SMN1 deletions in SMA families using a simple fluorescent multiplex PCR method. J. Med. Genet. 38: 240-243.

Schols L, Amoiridis G, Buttner T, Przuntek H, et al. (1997). Autosomal dominant cerebellar ataxia: phenotypic differences in genetically defined subtypes? Ann. Neurol. 42: 924-932.

Sequeiros J, Seneca S and Martindale J (2010). Consensus and controversies in best practices for molecular genetic testing of spinocerebellar ataxias. Eur. J. Hum. Genet. 18: 1188-1195.

Sobczak K and Krzyzosiak WJ (2005). CAG repeats containing CAA interruptions form branched hairpin structures in spinocerebellar ataxia type 2 transcripts. J. Biol. Chem. 280: 3898-3910. 